\title{
An Extremely Unusual Case of Type A Interrupted Aortic Arch in a Young Male
}

\author{
${ }^{1}$ Uma Debi, ${ }^{2}$ Prem Kumar, ${ }^{3}$ Manphool Singhal, ${ }^{4}$ Manoj Rohit
}

\begin{abstract}
A young male patient of 20-year-old came to our hospital for evaluation of hypertension in young. The patient has presented with symptoms of dizziness and heaviness of the head for past 3 months. ECHO was suggestive of coarctation of the aorta and was evaluated for the same with catheter angiography study. Aortograms were taken through both right brachial and femoral routes. Transfemoral catheter failed to pass beyond the proximal descending thoracic aorta, and flush angiograms showing aortic stump are taken. Transbrachial artery catheter angiogram showed abrupt cut off of the arch of aorta distal to the origin of the left subclavian artery. It was interpreted as severe coarctation of aorta and stent dilatation was planned during the same sitting. However even guide wire was not negotiable through the segment and procedure was abandoned. For proper delineation of aortic anatomy, multislice computed tomography (CT) angiography was performed which showed complete discontinuity of lumen of the aorta between the aortic arch and descending thoracic aorta which is separated by an atretic fibrous band of thickness $\sim 3.2 \mathrm{~mm}$ suggestive of interruption of the aorta (type A).
\end{abstract}

Keywords: Angiogram, Aorta, Aortic arch, Coarctation, Interrupted.

How to cite this article: Debi U, Kumar P, Singhal M, Rohit M. An Extremely Unusual Case of Type A Interrupted Aortic Arch in a Young Male. J Postgrad Med Edu Res 2019;53(1):31-33.

Source of Support: Nil

Conflict of Interest: None

\section{BACKGROUND}

Interruption of the arch of aorta is an extremely unusual congenital cardiovascular anomaly characterized by discontinuity of the lumen between the ascending and descending thoracic aorta. It represents less than $1 \%$ of congenital cardiac disease, and about $97 \%$ of these cases are associated with other cardiac anomalies. ${ }^{1}$ It differs from coarctation of aorta by its complete discontinuity of the lumen. This condition typically presents in the early neonatal period and carries a high risk of mortality if left untreated with a median age of death in untreated case $\sim 10$ days. ${ }^{1,2}$

\footnotetext{
${ }^{1}$ Assistant Professor, ${ }^{2}$ Senior Resident, ${ }^{3}$ Associate Professor, ${ }^{4}$ Professor

1-3 Department of Radiology and Imaging, Postgraduate Institute of Medical Education and Research, Chandigarh, India

${ }^{4}$ Department of Cardiology, Postgraduate Institute of Medical Education and Research, Chandigarh, India

Corresponding Author: Uma Debi, Assistant Professor, Postgraduate Institute of Medical Education and Research, Chandigarh, India, e-mail:Debi_uma@yahoo.co.in
}

\section{CASE REPORT}

A young male patient of 20-year-old came to our hospital for evaluation of hypertension in young. The patient has presented with symptoms of dizziness and heaviness of the head for past 3 months. ECHO was suggestive of the bicuspid aortic valve and mildly dilated aortic outlet diameter $(\sim 4.0 \mathrm{cms})$. On evaluation, patients blood pressure was found to be $100 / 80$. ECHO repeated in our center was suggestive of coarctation of the aorta and was evaluated for the same with catheter angiography study. Aortogram was taken through both right brachial and femoral routes. Transfemoral catheter failed to pass beyond the proximal descending thoracic aorta and flush angiograms showing aortic stump are taken. Transbrachial catheter angiogram showed abrupt cut off of the distal arch of the aorta just distal to the origin of the left subclavian artery. It was interpreted as severe coarctation of aorta and stent dilatation was planned during the same sitting. However even guide wire was not negotiable through the segment and procedure was abandoned.

For proper delineation of aortic anatomy, multislice CT Angiography was planned on 64 slice detector scanner (TOSHIBA). Multiplanar reconstructed and Volume rendered images were post-processed and evaluated on a Terracon workstation. Complete discontinuity of the aorta is seen between the aortic arch, and descending thoracic aorta was found which separated by an atretic fibrous band of thickness $\sim 3.2 \mathrm{~mm}$ suggestive of type A interrupted aortic arch. Extensive collaterals are seen pervading around this segment from anterior and posterior intercostal arteries seen supplying the descending thoracic aorta. Also, B/L internal thoracic arteries, dorsal scapular artery, and thoracoacromial artery are dilated and tortous with collaterals seen in the anterior chest wall, $\mathrm{B} / \mathrm{L}$ axillary region, and interscapular region.

Selected sections of magnetic resonance imaging (MRI) was planned for a demonstration of Interruption and bicuspid aortic valve using fast spin echo axial sequence and cine SSFP sequences in axial, candy cane view (sagittal oblique) and oblique view for the aortic valve.

\section{DISCUSSION}

Aortic arch interruption is a fatal and rare congenital anomaly was first described in $1778^{3}$ and was taken up for surgical repair for the first time in $1954 .{ }^{4}$ It is classified into three types by Celoria and Patton, ${ }^{5}$ depending upon the location of a discontinuity in the aortic arch: 

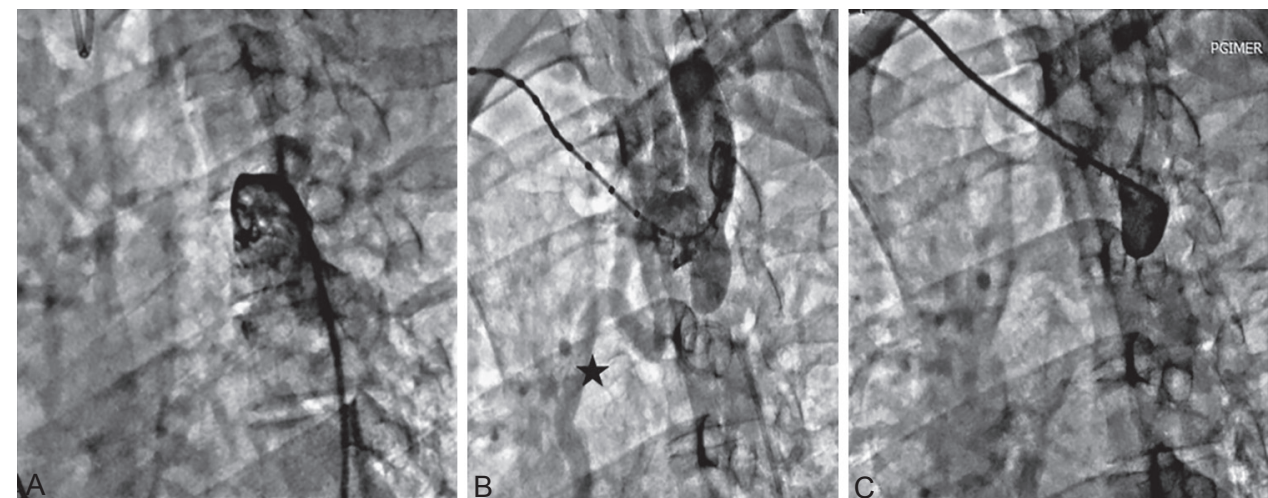

Figs $1 \mathrm{~A}$ to $\mathrm{C}$ : $(\mathrm{A})$ Contrast run through transfemoral catheter placed in proximal descending aorta shows non opacification of proximal aortic arch and; (B and C) that of transbrachial catheter placed in aortic arch shows non opacification of descending aorta. Dilated and tortous internal mammary artery can be seen (marked in star)

- Type A-Distal to the left subclavian artery

- Type B-Between the left common carotid and left subclavian arteries

- Type C-Between the brachiocephalic and left common carotid arteries

All these types again can be subdivided into subtype 1-normal subclavian artery; subtype 2-aberrant subclavian artery; and subtype 3-isolated subclavian artery arising from the ductus arteriosus.

The exact cause of interrupted aortic arch is still unknown with many theories in literature giving a hypothesis of abnormally decreased blood flow in the aorta in early embryological period causes interruption. ${ }^{6}$

Interruption is very rarely encountered in adult patients. To the best of our knowledge, there have been around 25 cases only reported in world literature ${ }^{7,8}$ indicating the rarity of this anomaly. In the few reported adult cases, clinical symptoms vary from normal to weakness, hypertension, malaise, headache, differences in blood pressures in the limbs, swelling of limbs and ischaemic changes in the lower limbs.

Type $\mathrm{A}$ is most common in presentation adults while neonates are most commonly presented with type B interruption. One review study in adults showed that around $\sim 80 \%$ of the interrupted aortic arch in adults is type A. ${ }^{8}$ Most of the patients will have associated cardiovascular anomalies. The most common associated anomaly in the adult presentation will be bicuspid aortic valve seen in more than $40 \%$ of these group of patients. Other cardiac common anomalies include PDA, VSD and aortopulmonary window seen in the commoner early infant presentation. PDA is the most common associated anomaly in children seen in around $\sim 97 \%$ of patients. ${ }^{9}$

Genetic abnormalities like CATCH22 mutation involving deletion of chromosome 22q11 ( which includes DiGeorge syndrome) are identified in $>80 \%$ of these group of patients indicating a causal association. ${ }^{9}$

Interruption can be evaluated with echocardiography, CT, and MRI. In a patient of suspected cardiac disease echocardiography is the first modality but it provides a suboptimal window for visualizing aortic arch, and descending thoracic aorta hence diagnosis of the interrupted aortic arch is not always accurate. Even distinction between Interruption, coarctation, and the hypoplastic arch is not always possible. Before surgery, the size of the major arteries (ascending aorta, the arch of the aorta and descending thoracic aorta) and branching pattern, the presence of bicuspid aortic vulve, the span of interruption, the presence and location of patent ductus arteriosus and other congenital heart disease need to be defined. Catheter angiography is an invasive technique, but it can evaluate all of the above and most of the time it may require arterial catheterizations at two different sites-via femoral route and brachial route as done in our case. It is also time-consuming with adjunct risks of a large amount of contrast injection and potential
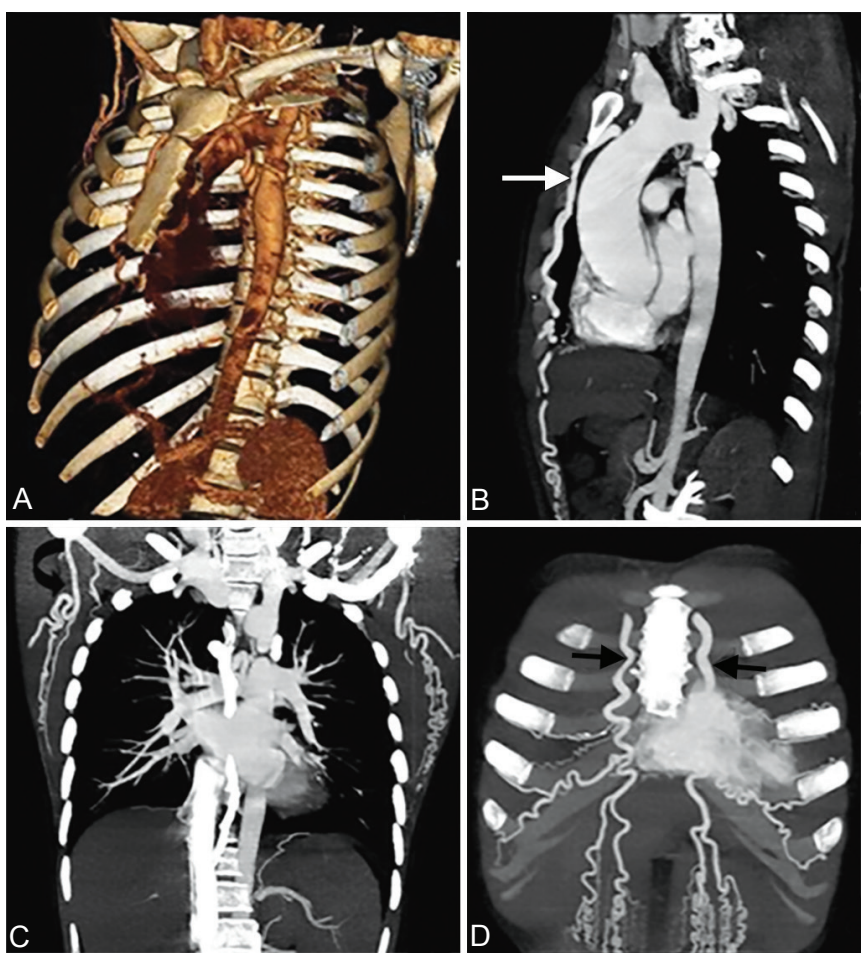

Figs 2A to D: (A) Volume rendered (VR) and; (B to D) (Multiplaner reconstructions) MPR of CT Angiography showing type A interruption with dilated and tortous internal mammary artery and dilated anterior intercostal arteries (marked in solid arrows) and thoracoacromial artery (marked in curved arrow) 

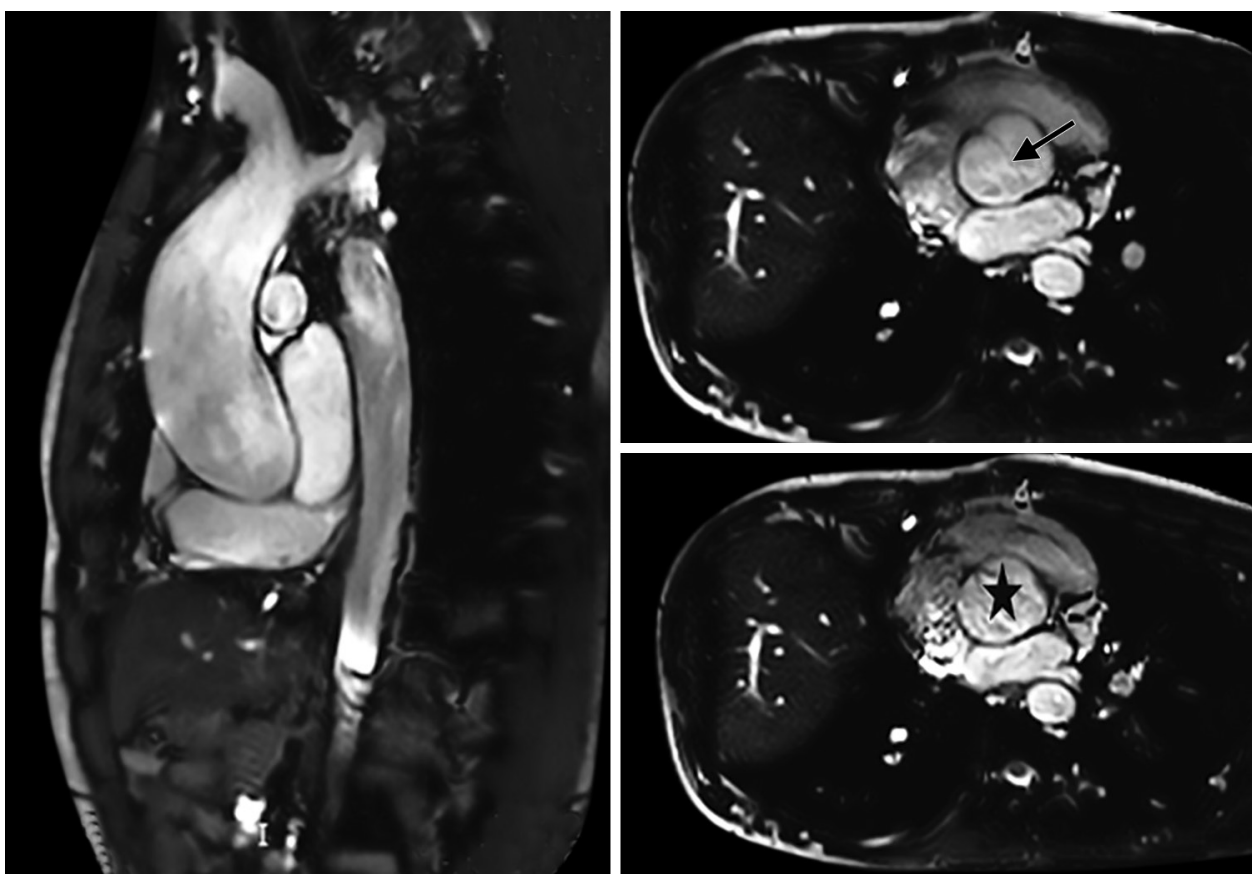

Figs 3 A to C: (A) Candy cane sagittal oblique SSFP cine sequence showing interrupted aortic arch; (B) Double oblique LVOT view SSFP sequence showing a single commissural line (arrow) of bicuspid aortic valve with right-left cusp orientation, a common associated condition noted in patients with interrupted aortic arch; (C) The fish mouth appearance of bicuspid aortic valve in systole with elliptical orifice (star)

complications of endovascular catheterization such as dissection and occlusion.

Multidetector CT angiography is a valuable investigation with reduced acquisition time as compared with other imaging modalities. It has a high spatial resolution as compared with angiography. Other advantages include multiplanar and 3D rendering, shorter procedure time and a wide range of coverage. MRI is also recently added for diagnosis of cardiac and vascular anomalies with contrast-enhanced MR angiography (CEMRA) reliable and effective method for diagnosis of complex cardiovascular abnormalities.

Interrupted aortic arch and Coarctation of aorta shares similar clinical features and imaging findings with complete luminal discontinuity seen in interruption differentiating it from coarctation. However, severe coarctation may present with similar radiological findings with cases showing the progression of coarctation into complete obstruction reported in the literature. ${ }^{10}$

The primary treatment modality offered to cases reported in the literature is surgery with most of them corrected in a single-stage repair. ${ }^{11}$ The first successful surgical repair was performed as early as 1961 in a 14-year child. ${ }^{12}$ Also, medical treatment with 4 years follow-up has been tried for in adult interruption of aorta presented with hypertension with the good collateral flow and has shown positive results with a focus on long term surveillance. ${ }^{13}$

\section{REFERENCES}

1. Reardon MJ, Hallman GL, Cooley DA. Interrupted aortic arch: brief review and summary of an eighteen-year experience. Texas Heart Institute Journal. 1984 Sep;11(3):250-259.
2. Bugan B, Iyisoy A, Celik M, Kucuk U, Boz U, Celik T. Isolated type A interrupted aortic arch in an asymptomatic 19-year-old man. Texas Heart Institute Journal. 2011;38(5):559-561.

3. Steidele RJ. Samml Chir u Med Beob. Vienna. 1778;2:114.

4. Gokcebay TM, Batillas J, Pinck RL. Complete interruption of the aorta at the arch. Am J Roentgenol 1972;114:362-370.

5. Celoria GC, Patton RB. Congenital absence of the aortic arch. American heart journal. 1959 Sep 1;58(3):407-413.

6. Dillman JR, Yarram SG, D'Amico AR, Hernandez RJ. Interrupted aortic arch: spectrum of MRI findings. American Journal of Roentgenology. 2008 Jun;190(6):1467-1474.

7. Sakellaridis T, Argiriou M, Panagiotakopoulos V, Krassas A, Argiriou O, Charitos C. Latent congenital defect: interrupted aortic arch in an adult-case report and literature review. Vascular and endovascular surgery. 2010 Jul;44(5):402-406.

8. John AS, Schaff HV, Drew T, Warnes CA, Ammash N. Adult presentation of interrupted aortic arch: Case presentation and a review of the medical literature. Congenital heart disease. 2011 May;6(3):269-275.

9. Sato S, Akagi N, Uka M, Kato K, Okumura Y, Kanazawa S. Interruption of the aortic arch: diagnosis with multidetector computed tomography. Japanese journal of radiology. 2011 Jan 1;29(1):46-50.

10. Vriend JW, Lam J, Mulder BJ. Complete aortic arch obstruction: interruption or aortic coarctation?. The international journal of cardiovascular imaging. 2004 Oct 1;20(5):393-396.

11. Messner G, Reul GJ, Flamm SD, Gregoric ID, Opfermann UT. Interrupted aortic arch in an adult: single-stage extra-anatomic repair. Texas Heart Institute Journal. 2002;29(2):118-121.

12. Villalobos MC, De Balderrama DP, y Lopez JL, Castellanos M. Complete interruption of the aorta. American Journal of Cardiology. 1961 Nov 1;8(5):664-669.

13. Öztürk A, Özcan EE, Özel E, Uyar S, enaslan Ö. Medical treatment of an adult with uncorrected isolated interrupted aorta resulted in no complications after 4 years of follow-up. The American journal of case reports. 2014;15:330-332. 